Changes included prolonged metabolic acidosis, decreased blood potassium, lower heart rate, and higher mean blood pressure, but were not associated with clinical complication. Unusual MRI findings in 3 infants were sinus thromboses and cerebral infarction. Six infants had normal follow-up neurologic exams or only minor abnormalities. Three infants died and 1 had major abnormalities. EEG activity returned to a more continuous pattern by 13 hours in 5 infants, although seizures recurred. EEG suppression was greater in infants with a poor outcome than in the 6 who recovered. Of 6 untreated infants with a normal initial EEG, none developed severe encephalopathy or neurologic sequelae. (Azzopardi D, Robertson NJ, Cowan FM et al. Pilot study of treatment with whole body hypothermia in neonatal encephalopathy. Pediatrics October 2000;106:684-694). (Reprints: Denis Azzopardi MD, FRCP, Department of Pediatrics, Imperial College School of Medicine, London, UK).

COMMENT. Prolonged mild hypothermia has been evaluated in the treatment of asphyxiated neonates at high risk of developing severe neonatal encephalopathy. A burst suppression pattern in the EEG is indicative of increased risk of severe encephalopathy and possible trial of hypothermia.

\title{
OUTCOME OF NEONATAL CEREBRAL INFARCTION
}

The long-term neurodevelopmental outcome of CT-documented cerebral infarction was evaluated in 46 children followed for a mean of 42 months (range, 18-164 months) at the Glenrose Rehabilitation Hospital, and University of Alberta, Edmonton, Canada. Outcome was normal in 15 and abnormal in 31 , with multiple disabilities in 23, cerebral palsy in 22, and cognitive impairment in 19. Risk factors for long-term disability were neonatal seizures and abnormal neurologic exam at discharge. (Sreenan C, Bhargava R, Robertson CMT. Cerebral infarction in the term newborn: clinical presentation and long-term outcome. I Pediatr September 2000;137:351-355). (Reprints: Charlene Robertson MD, FRCPC, Neonatal and Infant Follow-up Clinic, Glenrose Rehabilitation Hospital, 10230-111 Ave, Edmonton, Alberta, Canada T5G 0B7).

COMMENT. The long-term outcome in term neonates with cerebral infarction is normal in one third and abnormal in two thirds. Risk factors for disability are seizures and an abnormal neurologic examination at the time of discharge.

\section{MENTAL RETARDATION SYNDROMES}

\section{FRAGILE X SYNDROME WORKSHOP}

The proceedings of the 9 th International Workshop on Fragile X Syndrome and X-Linked Mental Retardation, held in Strasbourg, France, are reported from the University Hospital of Leuven, Belgium.

Several examples of X-Linked MR syndromes were presented.

Mohr-Tranebjaerg syndrome (MTS), characterized by deafness, dystonia and mental retardation (MR), was discussed as a mitochondrial disorder with a gene named DDP for deafness/dystonia peptide. Among a set of 8 patients with MTS, only one of 4 studied showed ragged-red fibers on muscle biopsy whereas $4 / 4$ had increased numbers of mitochondria.

In two papers on fragile $X$ syndrome (FXS), a total of 27 children ( 24 boys and 3 girls), the diagnosis had been missed by the referring physicians. Clinical manifestations were nonspecific, a positive family history was not considered, or 
an incorrect diagnosis had been made. The presenters made pleas for better education of professionals and a higher index of suspicion about FXS.

Psychologists proposed testing for patients with MR, using the Stanford Binet for IQ and the Vineland for Adaptive Behavior as a first stage, and areas of strength and weaknesses and more specific functions in second stage tests. Comparing cognitive and behavioral abilities in children with FXS and with nonFXS autism, IQ scores in FXS show a significant decline with time, whereas individuals with autism manifest relatively no change in cognitive levels. In contrast, both groups show significant longitudinal decreases in adaptive behavior. In another presentation, therapy of ADHD symptoms in 11 children with FXS, some showed an increase in performance on executive function tasks using Adderall. Other sessions on FXS were on population screening and the molecular basis of FXS. (Fryns J-P, Borghgraef M, Brown TW et al. Conference report. Am I Med Genet October 2000;94:345-360). (Respond: Jean-Pierre Fryns, Center for Human Genetics, Herestraat 49, B-3000 Leuven, Belgium).

COMMENT. The frequency of missed diagnosis in cases of FXS is of interest. Guidelines have previously been suggested to aid the practicing physician in determining which children should have a chromosomal analysis. (see Progress in Pediatric Neurology I, PNB Publishers, 1991;pp196-7).

An estimate of the prevalence of X-Linked MR syndromes is about 1:1000 mentally retarded males, 50\% having FXS. The clinical manifestations of FXS include: mental retardation, developmental delay, speech delay, ADHD, temper tantrums, mouthing of objects, autistic behaviors, and impaired gross motor coordination. ADHD is diagnosed in 50\% or more of FXS patients. Epilepsy occurs in $25 \%$. A positive family history for mental retardation is reported in $65 \%$, and $90 \%$ of cases have a family history of either retardation, learning disability, or hyperactivity.

Physical findings include: large ears (75\%), prominent jaw and long face (70\%), macrocephaly (40\%), hypertelorism (40\%), simian palmar creases (35\%), and large testicles (17\%). A chromosomal analysis for FX is most likely to be positive for FXS in young males with developmental delay (especially speech delay), a maternal family history for MR, and facial dysmorphism (especially large ears, long face, and prominent jaw). For further articles on FXS, see Progress in Pediatric Neurology II. PNB, 1994;295-299. Despite the guidelines and a high index of suspicion, particularly among boys with ADHD, I still find the selection of likely candidates for chromosome testing to be difficult, with few positives in my practice of pediatric neurology.

\section{CONGENITAL MALFORMATION SYNDROMES}

\section{GOMEZ-LOPEZ-HERNANDEZ SYNDROME: EXPANDED PHENOTYPE}

Since the first patient with cerebello-trigeminal-dermal dysplasia, later called Gomez-Lopez-Hernandez syndrome, was reported in 1979 by Gomez et al, six more cases have been added to the literature. Abnormalities involve the cerebellum (rhombencephalosynapsis), cranial nerves (trigeminal anesthesia), and scalp (alopecia). A further case is reported in a 19 year-old male seen at Tufts University School of Medicine, Boston, MA. The typical manifestations of the syndrome were present. In addition, some unusual findings include seizures, growth hormone deficiency and short stature, and prominent behavioral and psychiatric problems (hyperactivity, depression, self-injurious behavior, and bipolar disorder). Hyperactivity first noted at 4 years of age was later associated with attention deficit disorder. Chromosomal analysis was normal. (Brocks D, 